\title{
Light Neutralino Dark Matter in the pMSSM
}

\section{Implications of LEP, LHC and Dark Matter Searches on SUSY Particle Spectra}

\author{
A. Arbey $^{123}$, M. Battaglia ${ }^{345}$, and F. Mahmoudi ${ }^{36}$ \\ 1 Centre de Recherche Astrophysique de Lyon, Observatoire de Lyon, Saint-Genis Laval Cedex, F-69561, France; CNRS, UMR \\ 5574; Ecole Normale Supérieure de Lyon, Lyon, France \\ 2 Université de Lyon, France; Université Lyon 1, F-69622 Villeurbanne Cedex, France \\ 3 CERN, CH-1211 Geneva 23, Switzerland \\ 4 Santa Cruz Institute of Particle Physics, University of California, Santa Cruz, CA 95064, USA \\ ${ }^{5}$ Lawrence Berkeley National Laboratory, Berkeley, CA 94720, USA \\ ${ }^{6}$ Clermont Université, Université Blaise Pascal, CNRS/IN2P3, LPC, BP 10448, F-63000 Clermont-Ferrand, France
}

\begin{abstract}
We investigate the viability of light neutralino scenarios as promoted by dark matter direct detection experiments. Using high statistics scans in the pMSSM we have identified several scenarios which give rise to very light neutralinos with large direct detection scattering cross sections. Our results are challenged with constraints from dark matter relic density, direct detection, indirect detection, as well as flavour physics, electroweak precision tests, LEP and Tevatron limits, LHC limits on SUSY, Higgs and monojet searches. In particular we study the effect of a Higgs boson in the range $122.5<M_{h}<127.5$ $\mathrm{GeV}$. We show that several scenarios emerge in agreement with all the constraints, and we study their characteristics and the LHC sensitivity.
\end{abstract}

PACS. 11.30.Pb Supersymmetry - 14.80.Da Supersymmetric Higgs bosons - 14.80.Nb Neutralinos and charginos - 95.35.+d Dark matter

\section{Introduction}

One of the appealing features of Supersymmetry (SUSY), which has contributed to promote it to the role of template model of new physics beyond the Standard Model, is the natural inclusion of a weakly interacting massive particle (WIMP) as cold dark matter (DM) candidate. In most of the cases, this is the lightest neutralino, $\tilde{\chi}_{1}^{0}$, which is the lightest supersymmetric particle (LSP). Scenarios with neutralino LSP realise the exact amount of dark matter relic density to match the precise determination obtained in the study of cosmic microwave background $(\mathrm{CMB})$. This happens for neutralino masses of $\mathcal{O}(100 \mathrm{GeV})$ due to the suggestive fact that a particle with $\sim 100 \mathrm{GeV}$ mass and typical weak interaction couplings gives the correct neutralino relic density, $\Omega_{\mathrm{CDM}} h^{2} \sim 0.1$, in standard cosmology models which often referred to as the "WIMP miracle".

The analysis of constraints from low energy data, dark matter density and the results of the LHC searches in generic SUSY scenarios shows that light neutralinos are still well compatible with current data. While searches for signals of strongly interacting SUSY particles in $p p$ collisions using missing transverse energy (MET) signatures are ongoing at the LHC, ground-based direct detection DM experiments, such as XENON [1] and CDMS [2], have reached a sensitivity in spin-independent WIMP-nucleon cross section at WIMP masses of $\mathcal{O}(100 \mathrm{GeV})$, which covers a significant fraction of the SUSY parameter space explored at the LHC. The present XENON-100 limit [1] covers $\sim 20 \%$ of the MSSM parameter space compatible with low energy data and the relic dark matter constraint 3 .

The possibility that the LSP mass is much smaller than the electroweak scale, is put forward by three dark matter direct detection experiments, which have reported a possible signal of WIMP interaction corresponding to very light particles, $5<M_{\mathrm{CDM}}<15 \mathrm{GeV}$ close to the edge of the XENON-100 and CDMS sensitivity. These are the DAMA experiment [4, 5] at the Laboratory Nazionali del Gran Sasso, Italy, the CoGENT experiment 6, 7] in the Soudan mine and the CRESST experiment [8, also at Gran Sasso. While there is still substantial debate on the interpretation of these data and the compatibility of the reported results with the exclusion bounds established by the CDMS and XENON experiments 912 an agreement could only be possible in the low neutralino mass region. Therefore it is interesting to explore the feasibility of light neutralino solutions in generic SUSY models and their compatibility with the results of the LEP and LHC searches.

In this study we consider SUSY scenarios with the lightest neutralino mass in the range $5<M_{\chi_{1}^{0}}<40 \mathrm{GeV}$ in the 19-parameter pMSSM model. The pMSSM is a 
minimal supersymmetric extension of the Standard Model (MSSM) with $R$-parity conservation, where the masses of all SUSY particles are treated as free parameters [13. The use of the pMSSM enables us to access scenarios which are not available in constrained SUSY models, such as the CMSSM.

There have been already several studies of the SUSY parameter space with light neutralinos 14 30. They considered either a constrained MSSM scenario or more generic analyses in effective MSSM scenarios where gaugino masses are not unified at the GUT scale. In addition, analyses within the NMSSM scenarios were also performed [27, 3135. Compared to those studies we consider here a broader phase space of parameters and study for the first time the light neutralinos in the pMSSM in its full glory with 19 free parameters. In particular, we do not assume degenerate masses for the right and left handed squarks, contrary to the previous works. By this choice, different squark mixings are allowed and scenarios with light squarks and reduced couplings to the $Z^{0}$ boson can be realised in our scans of the parameter space. This study reveals therefore scenarios not yet identified in the earlier works. We employ a large statistics of more than $500 \mathrm{M}$ pMSSM points for a generic scan and more than $500 \mathrm{M}$ extra points for specific scans, and impose the latest constraints from the LHC data, including those from Higgs and monojet searches. In particular we study the effect of a Higgs boson in the range $122.5<M_{h}<127.5 \mathrm{GeV}$. We use a realistic simulation, validated on the results of full simulation and reconstruction to study the phenomenology of these scenarios and the response of the standard SUSY searches on $5 \mathrm{fb}^{-1}$ of data at $7 \mathrm{TeV} 36$.

This paper is organised as follows. Section 2 describes the region highlighted by the results of the DAMA, CoGENT and CRESST direct dark matter searches and the pMSSM simulation with the constraints applied to reproduce bounds from flavour physics, electroweak data, earlier SUSY searches at LEP-2 and the Tevatron and dark matter relic density. The different viable pMSSM scenarios with a light neutralino are presented. Section 3 discusses their phenomenology at the LHC and the impact of the SUSY searches based on jets, leptons and missing transverse energy. Section 4 has the conclusions.

\section{The pMSSM Phase Space with light neutralino LSP}

\subsection{Simulation and Tools}

The study of the pMSSM parameter space with light neutralinos is based on the combination of several programs from spectrum generation to the computation of dark matter scattering cross sections and relic density, flavour and electroweak observables, as well as the simulation and analysis of events in $7 \mathrm{TeV} p p$ collisions. This study is part of a broad program on the implications of LHC results for the MSSM through scans of the pMSSM. A detailed description of the software tools employed is given

\begin{tabular}{|c|c|}
\hline Parameter & Range \\
\hline \hline $\tan \beta$ & {$[1,60]$} \\
\hline$M_{A}$ & {$[50,2000]$} \\
\hline$M_{1}$ & {$[-300,300]$} \\
\hline$M_{2}$ & {$[0,2500]$} \\
\hline$M_{3}$ & {$[-10000,10000]$} \\
\hline$A_{d}=A_{s}=A_{b}$ & {$[-10000,10000]$} \\
\hline$A_{u}=A_{c}=A_{t}$ & {$[-10000,10000]$} \\
\hline$A_{e}=A_{\mu}=A_{\tau}$ & {$[-3000,3000]$} \\
\hline$\mu$ & {$[0,2500]$} \\
\hline$M_{\tilde{e}_{L}}=M_{\tilde{\mu}_{L}}$ & {$[0,2500]$} \\
\hline$M_{\tilde{e}_{R}}=M_{\tilde{\mu}_{R}}$ & {$[0,2500]$} \\
\hline$M_{\tilde{\tau}_{L}}$ & {$[0,2500]$} \\
\hline$M_{\tilde{\tau}_{R}}$ & {$[0,2500]$} \\
\hline$M_{\tilde{q}_{1 L}}=M_{\tilde{q}_{2 L}}$ & {$[0,2500]$} \\
\hline$M_{\tilde{q}_{3 L}}$ & {$[0,2500]$} \\
\hline$M_{\tilde{u}_{R}}=M_{\tilde{c}_{R}}$ & \\
\hline$M_{\tilde{t}_{R}}$ & {$[00]$} \\
\hline$M_{\tilde{d}_{R}}=M_{\tilde{s}_{R}}$ & {$[0,2500]$} \\
\hline$M_{\tilde{b}_{R}}$ & {$[000]$} \\
\hline
\end{tabular}

Table 1. pMSSM parameter ranges adopted in the scans (in $\mathrm{GeV}$ when applicable).

in 36. We mention here only the software tools most relevant to this study. SUSY spectra are generated with SOFTSUSY 3.2.3 37. The widths and decay branching fractions of SUSY particles are computed using SDECAY 1.3 [38. The dark matter relic density is calculated with SuperIso Relic v3.2 39, 40, which provides us also with the flavour observables. MicrOMEGAs 2.4 [41] is used to compute neutralino-nucleon scattering cross-sections. Corrections to the $Z^{0}$ electroweak observables are calculated analytically. We perform flat scans of the 19 pMSSM parameters within the ranges summarised in Table 1. For this study, over one billion pMSSM points have been generated in total.

Event generation of inclusive SUSY samples in $e^{+} e^{-}$ and $p p$ collisions is performed with PYTHIA 6.4.24 42. Cross sections for $p p$ collisions are rescaled to their NLO values by the k-factors obtained with Prospino 2.0 [43. Samples of $e^{+} e^{-}$events are reconstructed at generator level to assess their observability at LEP-2. Hadronic jets are clustered using the PYCLUS algorithm with $d_{\text {join }}=$ $2.5 \mathrm{GeV}$. LHC generated events are passed through fast detector simulation using Delphes 1.9 [44] tuned for the CMS detector. The event reconstruction follows the procedure of the CMS SUSY analyses as discussed in [36].

\subsection{Constraints}

To constrain the pMSSM parameter space, we apply different limits, from cosmological data, flavour physics, electroweak data, and collider searches. 


\subsubsection{Dark Matter}

Dark matter constraints arise from the relic density determination, $\Omega_{\mathrm{CDM}} h^{2}$, mostly from the analysis of the WMAP data [45, and from direct detection experiments. For the relic density, we consider two intervals: the tight WMAP bound of $0.068<\Omega_{\chi} h^{2}<0.155$ which includes the theoretical uncertainties. We study also the effects of a loose limit where we request that the neutralino contribution, $\Omega_{\chi} h^{2}$, is non zero and below, or equal to, the upper limit set by the WMAP result: $10^{-4}<\Omega_{\chi} h^{2}<$ 0.155 . This leaves the possibility of other sources of dark matter alongside the lightest neutralino. In section 2.4. where we discuss non-standard scenarios, we further relax this requirement.

The results of dark matter direct detection experiments are more problematic. The claims from the experiments reporting an excess of events compatible with the signal of a light WIMP must be confronted with the limits obtained by XENON [1] and CDMS [2, which has also performed analyses relaxing the energy cut-off [46] and, more recently, searched for an annual modulation of the event rate 47. In this study we accept pMSSM with 5 $\leq M_{\chi_{1}^{0}}<40 \mathrm{GeV}$ and $10^{-7}<\sigma_{\chi p}^{\mathrm{SI}}<10^{-3} \mathrm{pb}$, in order to be in the region where data could be reconciled.

Other cosmological bounds can come from indirect detection signatures, but they are subject to large cosmological and astrophysical uncertainties. We do not impose them as a constraints in the analysis but we comment on the consequences of the Fermi-LAT results 48 in Section 2.3 .

\subsubsection{Flavour Physics}

Flavour physics sets important constraints on the SUSY parameters. We impose bounds from $b$ and $c$ meson decays, which have been discussed in details in 36. In particular, the decay $B_{s}^{0} \rightarrow \mu^{+} \mu^{-}$, which can receive extremely large SUSY contributions at large $\tan \beta$, deserves special attention 49. An excess of events in this channels was reported by the CDF-II collaboration at the Tevatron 50 and upper limits have been set by the LHCb 51 and CMS 52 collaborations at LHC. Recently the LHCb collaboration has presented their latest result for the search of this decay based on $1 \mathrm{fb}^{-1}$ of data. A $95 \%$ C.L. upper limit on its branching fraction is set at $4.5 \times 10^{-9}$ [51, which closely approaches the SM prediction of $(3.53 \pm$ $0.38) \times 10^{-9}$ for the rate of this process [53]. After accounting for theoretical uncertainties, estimated at the $10 \%$ level, the constraint $\operatorname{BR}\left(B_{s}^{0} \rightarrow \mu^{+} \mu^{-}\right)<5 \times 10^{-9}$ is used in this analysis.

\subsubsection{Electroweak data}

The precision $Z^{0}$ line-shape and other electroweak observables place serious constraints on new particles. In particular, the accurate measurements of the $Z^{0}$ total width and its partial decay widths obtained at LEP provide a tight bound to the contribution from new particles with mass below $M_{Z^{0}} / 2$. The scenarios considered here with light $\chi_{1}^{0}$ are constrained from the neutralino contribution to the $Z^{0}$ invisible width. We compute the SM $Z^{0}$ total width and that into neutrinos using ZFitter 6.42 [54] for the input parameter ranges $115<M_{H}<145 \mathrm{GeV}, M_{\text {top }}=$ $(172.9 \pm 1.1) \mathrm{GeV}$ and $\alpha_{s}\left(M_{Z}^{2}\right)=0.1184 \pm 0.0007$. We obtain a SM $Z^{0}$ total width $\Gamma_{\text {tot }}=(2494.83 \pm 0.54) \mathrm{MeV}$ and invisible width $\Gamma_{\mathrm{inv}}=(501.62 \pm 0.10) \mathrm{MeV}$, where the uncertainty reflects the range of values used for the input parameters. These have to be compared to the average of LEP measurements giving $(2495.2 \pm 2.3)$ and $(499.0 \pm 1.5)$ $\mathrm{MeV}$, respectively. We excludes light neutralinos with a contribution to the $Z^{0}$ invisible width larger than $3 \mathrm{MeV}$. This restricts the acceptable points to those where the $\tilde{\chi}_{1}^{0}$ is bino-like and its contribution to the $Z^{0}$ width, $\Gamma_{\chi}$, is negligible so it can evade the LEP electroweak bounds and corresponds to relatively large values of the higgsino mass parameter $|\mu|$.

The $Z^{0}$ total width constrains the masses of the lightest chargino, $\tilde{\chi}_{1}^{ \pm}$, and of the squarks of the first two generations, $\tilde{q}$, to be above $45 \mathrm{GeV}$. Squarks of the third generation can evade these constraints for specific values of their $\theta_{\tilde{q}}$ angle in the mass mixing matrix, corresponding to a vanishing $Z^{0}$ coupling, $I_{3}^{q} \cos ^{2} \theta_{\tilde{q}}-Q_{\tilde{q}} \sin ^{2} \theta_{W}$. We also compute the $\tilde{b_{1}} \tilde{\tilde{b}}_{1}$ contribution to the $Z^{0}$ width for each generated pMSSM points and require it to be smaller than $5 \mathrm{MeV}$, to satisfy the hadronic $Z^{0}$ width limits.

\subsubsection{SUSY searches at LEP-2 and Tevatron}

The general constraints on SUSY particle masses from direct searches at lower energy colliders are summarised in Table 2. However, we note that constraints from SUSY searches at LEP-2 and Tevatron can be evaded in case of small mass splittings with the lightest neutralino, corresponding to low energy, or transverse energy, released in the detector or to vanishing couplings. Since these are crucial for assessing the viability of several of the small mass splitting solutions highlighted in this study, we explicitly study the detectability of the points fulfilling our selection criteria. For each point we generate an inclusive SUSY sample of 10k events in $e^{+} e^{-}$collisions at $\sqrt{s}=$ $208 \mathrm{GeV}$ and apply the LEP-2 reconstruction and selection criteria of the analyses of [55]. First, we consider the $\chi_{1}^{+} \chi_{1}^{-}$and $\chi_{2}^{0} \chi_{1}^{0}$ channels and adopt the SM backgrounds estimated in the original analyses for these channels. We compute the cross sections and the number of signal events passing the selection criteria. We compare these cross sections to the minimum excluded value as a function of $M_{\chi_{1}^{ \pm}}$ and $\Delta M=M_{\chi_{1}^{ \pm}}-M_{\chi_{1}^{0}}$ from the final combination of the LEP-2 results. We reject points which give a cross section in excess to that excluded by the combined LEP-2 data for the same $\Delta M$ and a number of selected signal events larger than the SM background. A first estimate indicates that chargino masses smaller than $40 \mathrm{GeV}$ are excluded by LEP data, independently from $\Delta M$. Therefore, we impose the constraint $M_{\chi_{1}^{ \pm}}>40 \mathrm{GeV}$ in the following, and 


\begin{tabular}{|c|c|c|}
\hline Particle & Limits & Conditions \\
\hline \hline$\tilde{\chi}_{2}^{0}$ & 62.4 & $\tan \beta<40$ \\
\hline$\tilde{\chi}_{3}^{0}$ & 99.9 & $\tan \beta<40$ \\
\hline$\tilde{\chi}_{4}^{0}$ & 116 & $\tan \beta<40$ \\
\hline$\tilde{\chi}_{1}^{ \pm}$ & 92.4 & $m_{\tilde{\chi}_{1}^{ \pm}}-m_{\tilde{\chi}_{1}^{0}}<4 \mathrm{GeV}$ \\
& 103.5 & $m_{\tilde{\chi}_{1}^{ \pm}}-m_{\tilde{\chi}_{1}^{0}}>4 \mathrm{GeV}$ \\
\hline$\tilde{e}_{R}$ & 73 & \\
\hline$\tilde{e}_{L}$ & 107 & $m_{\tilde{\tau}_{1}}-m_{\tilde{\chi}_{1}^{0}}>15 \mathrm{GeV}$ \\
\hline$\tilde{\tau}_{1}$ & 81.9 & $m_{\tilde{u}_{R}}-m_{\tilde{\chi}_{1}^{0}}>10 \mathrm{GeV}$ \\
\hline$\tilde{u}_{R}$ & 100 & $m_{\tilde{u}_{L}}-m_{\tilde{\chi}_{1}^{0}}>10 \mathrm{GeV}$ \\
\hline$\tilde{u}_{L}$ & 100 & $m_{\tilde{t}_{1}}-m_{\tilde{\chi}_{1}^{0}}>10 \mathrm{GeV}$ \\
\hline$\tilde{t}_{1}$ & 95.7 & $m_{\tilde{d}_{R}}-m_{\tilde{\chi}_{1}^{0}}>10 \mathrm{GeV}$ \\
\hline$\tilde{d}_{R}$ & 100 & $m_{\tilde{d}_{L}}-m_{\tilde{\chi}_{1}^{0}}>10 \mathrm{GeV}$ \\
\hline$\tilde{d}_{L}$ & 100 & $m_{\tilde{\chi}_{1}^{0}}<70 \mathrm{GeV}, m_{\tilde{b}_{1}}-m_{\tilde{\chi}_{1}^{0}}>30 \mathrm{GeV}$ \\
& 248 & $80 \mathrm{GeV}, m_{\tilde{b}_{1}}-m_{\tilde{\chi}_{1}^{0}}>30 \mathrm{GeV}$ \\
& 220 & $m_{\tilde{\chi}_{1}^{0}}<80 \mathrm{GeV}_{1} \mathrm{GeV}_{\tilde{b}_{1}}-m_{\tilde{\chi}_{1}^{0}}>30 \mathrm{GeV}$ \\
$\tilde{b}_{1}$ & 210 & $m_{\tilde{\chi}_{1}^{0}}<100 \mathrm{GeV}, m_{\tilde{b}_{1}}-m_{\tilde{\chi}_{1}^{0}}>30 \mathrm{GeV}$ \\
& 200 & $m_{\tilde{\chi}_{1}^{0}}<105 \mathrm{GeV}, m_{\tilde{\chi}_{1}}$ \\
\hline$\tilde{g}$ & 100 & $m_{\tilde{b}_{1}}-m_{\tilde{\chi}_{1}^{0}}>5 \mathrm{GeV}$ \\
\hline
\end{tabular}

Table 2. Constraints on the SUSY particle masses (in $\mathrm{GeV}$ ) from searches at LEP and the Tevatron

we will analyse a posteriori the exclusion of the points passing all the other constraints.

Then, we consider the $\tilde{b}_{1} \overline{\tilde{b}}_{1}$ channel for small $\Delta M=$ $M_{\tilde{b}_{1}}-M_{\chi_{1}^{0}}$ values. We simulate $e^{+} e^{-} \rightarrow \tilde{b}_{1} \overline{\tilde{b}}_{1}$ at $\sqrt{s}=$ $208 \mathrm{GeV}$ for each point fulfilling our cuts. Again, we compare the production cross section to the minimum value excluded by the combination of the results of the LEP-2 experiments as a function of $\Delta M$ and reject points with cross sections in excess to this value.

\subsubsection{Higgs and SUSY searches at LHC}

The searches conducted by ATLAS and CMS on the $7 \mathrm{TeV}$ data have already provided a number of constraints relevant to this study. First, the MET analyses [56, 57] have excluded a fraction of the MSSM phase space corresponding to gluinos below $\sim 600 \mathrm{GeV}$ and scalar quarks of the first two generations below $\sim 400 \mathrm{GeV}$. These are included using the same analysis discussed in [36, extended to an integrated luminosity of $5 \mathrm{fb}^{-1}$. Then, the search for the $A^{0} \rightarrow \tau^{+} \tau^{-}$decay [58, 59] has excluded a significant fraction of the $M_{A}-\tan \beta$ plane at low values of $M_{A}$ and large to intermediate values of $\tan \beta$.

But the LHC 2011 data has not only brought exclusions. The excess of events on the $\gamma \gamma$, four-lepton and $\ell \nu \ell \nu$ final states reported by both ATLAS [60] and CMS 61. provide an intriguing hint for a Higgs boson. If these events are the first signal of a boson with mass $M_{h} \simeq 125 \mathrm{GeV}$, they will represent a significant constraints on our scenarios, in particular for their implication on the scalar top mass 62. Here, we require $122.5<M_{h}<127.5 \mathrm{GeV}$, which corresponds to the mass region allowed by the current data.

Finally, the results of the searches for monojets and isolated photons 63 can be used to set constraints on the quark-neutralino couplings and thus on the $\chi p$ scattering cross-sections 64. In particular, the constraints derived for the spin-dependent cross-sections are more severe than those from direct detection experiments. Contrary to dark matter direct searches, the collider limits do not depend on astrophysical assumptions, such as the inferred local density of dark matter, which suffers from large uncertainties.

In this analysis we apply the constraints on both spindependent and spin-independent $\sigma_{\chi p}$ from the LHC searches to our pMSSM points.

\subsection{Allowed Regions}

We select the valid pMSSM points fulfilling the requirements discussed above; in particular the light neutralino mass in the range $5<M_{\tilde{\chi}_{1}^{0}}<40 \mathrm{GeV}$, relic dark matter density as given in section 2.2.1 and spin-independent scattering cross-section $10^{-7}<\sigma_{x p}^{\mathrm{SI}}<10^{-3} \mathrm{pb}$.

In general, for such a light LSP the relic density is larger than the upper relic density constraint, as shown in Fig. 1. However, if the mass difference between the next lightest supersymmetric particle (NLSP) and the LSP, $\Delta M$, is small enough, the relic density is strongly decreased by the coannihilations of the two particles in the early universe and $\Omega_{\chi} h^{2}$ can even fall below the lower limit applied for this study. pMSSM points compatible with the relic density constraint have values of the $\Delta M$ mass splitting of just a few GeV. The situation for the scattering cross-section is reversed: most of the selected pMSSM points have small scattering cross-sections, as shown in Fig. 2. In order to increase it up to the values highlighted by the direct search experiments claiming a light WIMP signal, $\sigma_{\chi p}^{\mathrm{SI}} \sim 10^{-6} \mathrm{pb}$, we have to require $\Delta M$ values below $1 \mathrm{GeV}$, which corresponds to a requirements opposite to that found for the relic density. Therefore, we can respect all the constraints imposed on the pMSSM points with light neutralinos only for few, very specific scenarios.

The effect of the collider data, SUSY and monojet searches, flavour and electroweak constraints, on the pMSSM points generated with our scan is shown in Fig. 3. The collider constraints decrease the number of points without significantly modifying their distribution in the $M_{\tilde{\chi}_{1}^{0}}$ $-\sigma_{\chi p}^{S I}$ space. However, the electroweak constraints have a significant impact in removing points at large scattering cross-sections, especially for larger values of the neutralino mass. The constraints from Higgs searches alone is shown in Fig. 4. Again, the Higgs mass constraint reduces the number of points, but does not modify their overall distribution. Using the points from the generic pMSSM scan, we find 20 and 5 points passing all the selections in the region of interest, for the loose and tight WMAP constraints, respectively. The effect of the various selection criteria on 

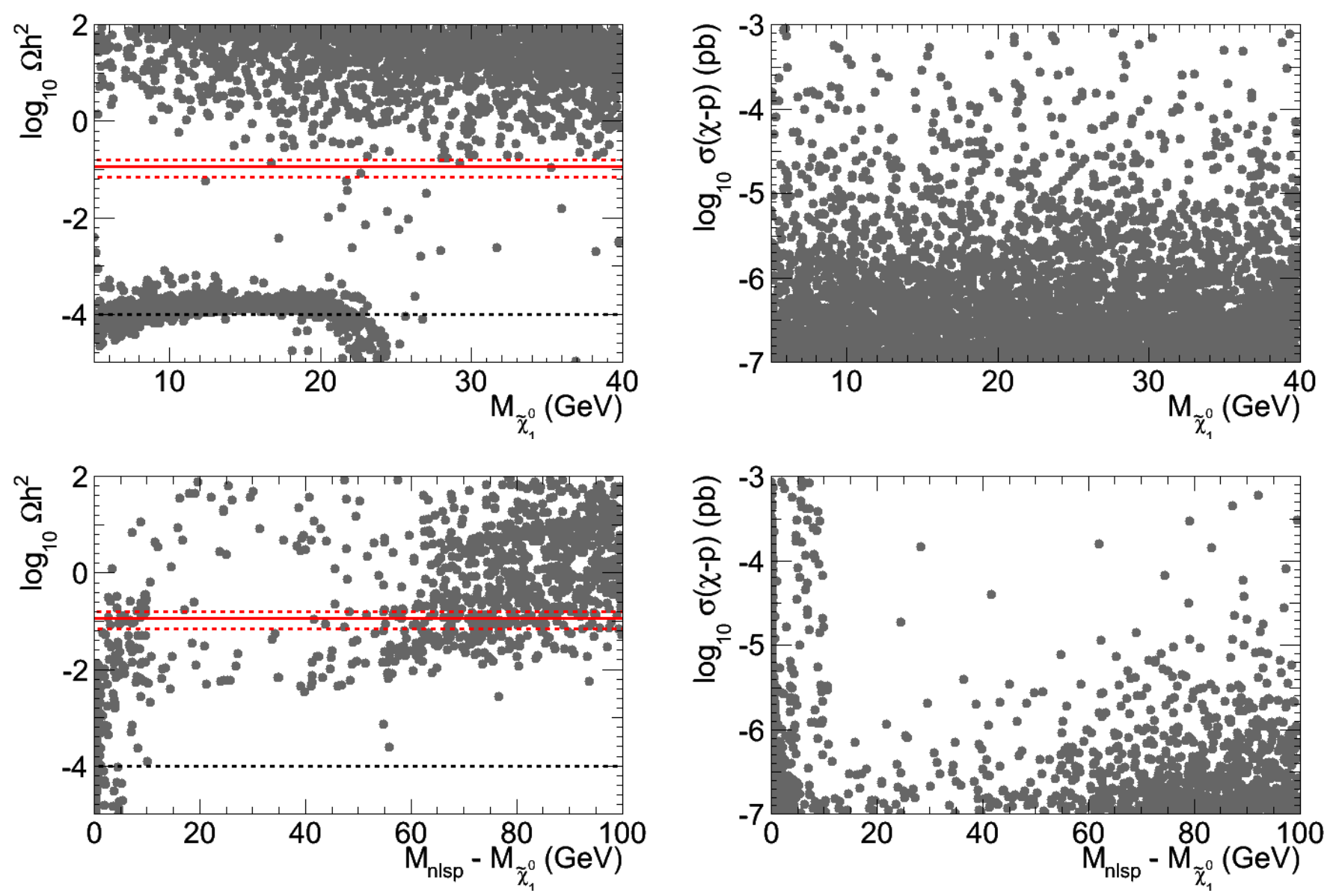

Fig. 1. Relic density in function of the $\tilde{\chi}_{1}^{0}$ mass (upper panel) and NLSP mass (lower panel). All the points in these plots pass the constraints described in section 2.2. The continuous red line corresponds to the WMAP dark matter central values, and the dashed red lines to the upper and lower limits that we impose. The dashed black line corresponds to the lower limit that we use when relaxing the lower WMAP constraint.

the number of scan points retained is summarised in Table 3. The relic density constraint in this region of the parameter space is rejecting a particularly large fraction of pMSSM points. This underscores the difficulty to harmonise the large scattering cross-section corresponding to the possible light WIMP signals and the WMAP results discussed above. In order to improve the statistics for specific scenarios, we perform specific scans within restricted parameter sets starting from the points passing the relic density constraints. We identify three distinct classes of pMSSM solutions: i) the NLSP is a slepton slightly above the LEP limit, with a neutralino of about $30 \mathrm{GeV}$ ii) the lightest chargino is degenerate with the $\tilde{\chi}_{1}^{0}$, often with a compressed gaugino spectrum and light Higgs bosons and iii) a scalar quark is degenerate with the $\tilde{\chi}_{1}^{0}$ while other scalar quarks and leptons are relatively heavy. Given the Higgs mass constraints, the possible light squarks for class iii) are those of the first and second generations or the lightest scalar bottom quark, $\tilde{b}_{1}$.

Fig. 2. Spin independent $\chi-p$ scattering cross-section as a function of the $\tilde{\chi}_{1}^{0}$ mass (upper panel) and NLSP mass (lower panel). All the points in these plots pass the constraints described in section 2.2

\begin{tabular}{|l|c|c|c|}
\hline Selection & $\begin{array}{c}\text { pMSSM } \\
\text { points }\end{array}$ & $\begin{array}{c}\text { Selection } \\
\text { Efficiency }\end{array}$ & $\begin{array}{c}\text { Cumulative } \\
\text { Efficiency }\end{array}$ \\
\hline \hline $\begin{array}{l}\text { Valid points with } \\
\text { light } \chi_{1}^{0}, \text { large } \sigma(\chi-p)\end{array}$ & $1 \mathrm{M}$ & - & - \\
\hline Monojet searches & $280 \mathrm{k}$ & 0.28 & 0.28 \\
\hline SUSY searches & $90 \mathrm{k}$ & 0.33 & 0.09 \\
\hline LEP searches & $50 \mathrm{k}$ & 0.60 & 0.05 \\
\hline Flavour physics & $20 \mathrm{k}$ & 0.37 & 0.02 \\
\hline Higgs searches & $10 \mathrm{k}$ & 0.47 & 0.01 \\
\hline Loose WMAP limit & 20 & $2 \times 10^{-3}$ & $2 \times 10^{-5}$ \\
\hline Tight WMAP limit & 5 & 0.25 & $5 \times 10^{-6}$ \\
\hline
\end{tabular}

Table 3. Scan statistics for the generic scan.

\subsection{1 $\tilde{\ell} \mathrm{NLSP}$}

We consider here the case of points with slepton NSLP. An example of a viable mass spectrum is given in Fig. 5. Since the mass limits from LEP-2 are higher for left-handed sleptons, right-handed sleptons are favoured by the relic density constraint, which requires a small splitting. Beyond the small mass of the NLSP sleptons, this scenario re- 


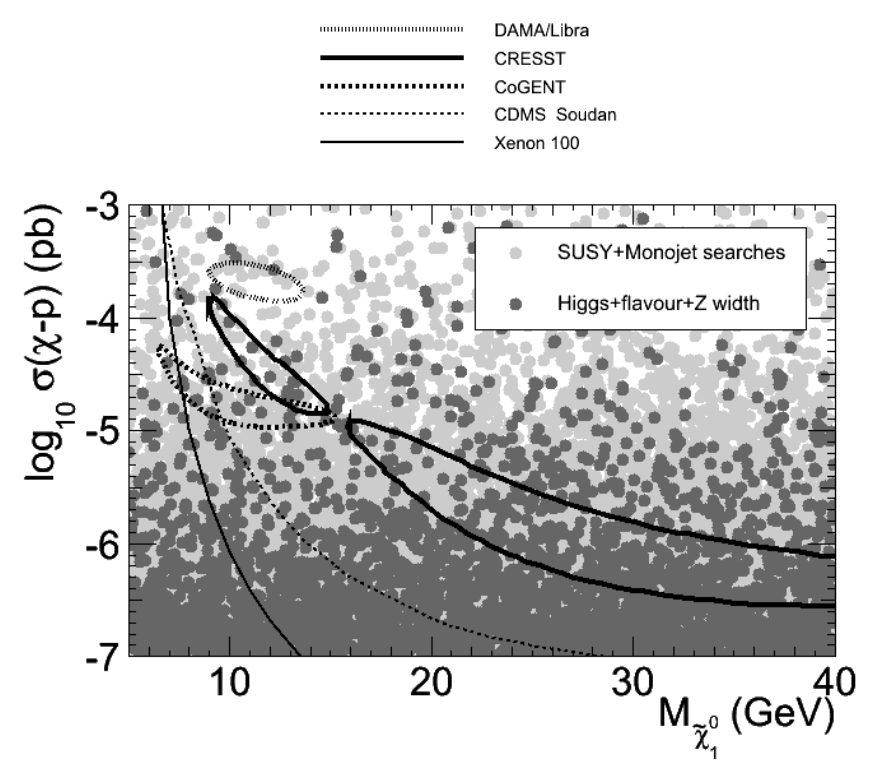

Fig. 3. Spin independent $\chi-p$ scattering cross-section as a function of the $\tilde{\chi}_{1}^{0}$ mass. The light gray points are consistent with the direct and monojet search limits (Higgs searches excluded). The dark gray points pass the constraints from flavour physics, the $Z$ decay constraints, and the Higgs mass limit $122.5<M_{h}<127.5 \mathrm{GeV}$. The thick contour lines correspond to the zones favoured by DAMA, CRESST and CoGENT, and the thin lines to the exclusion limits by CDMS and XENON.

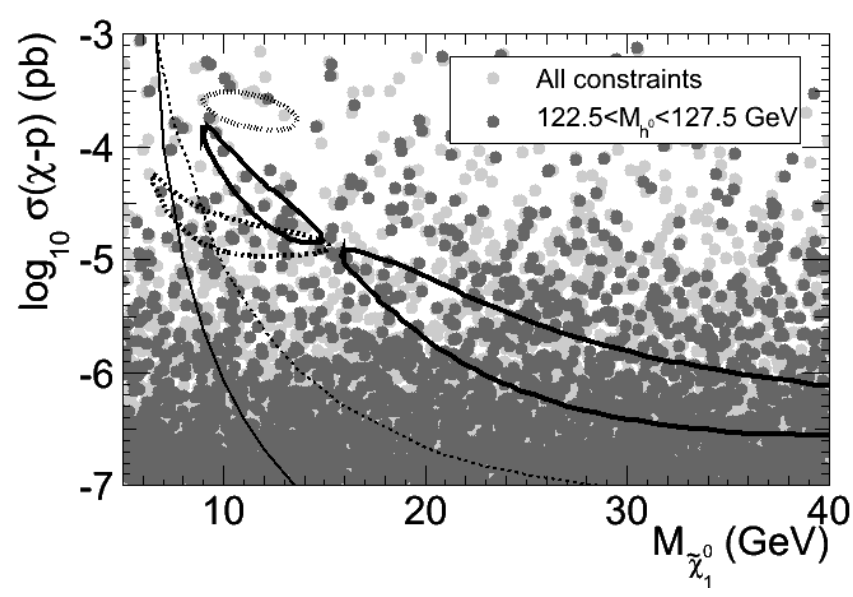

Fig. 4. Spin independent $\chi-p$ scattering cross-section as a function of the $\tilde{\chi}_{1}^{0}$ mass. The light gray colour corresponds to the points passing all the constraints presented in Fig. 3. except the Higgs mass limit $122.5<M_{h}<127.5 \mathrm{GeV}$. The dark gray points also pass the Higgs mass limits. The contour lines are the same as in Fig. 3

mains relatively standard. However, a neutralino mass of about at least $20 \mathrm{GeV}$ is needed to accommodate the upper relic density bound, as can be seen from Fig. 1. since the $\Delta M$ splitting remains relatively large. Therefore, this scenario has only a limited interest corresponding to a re-

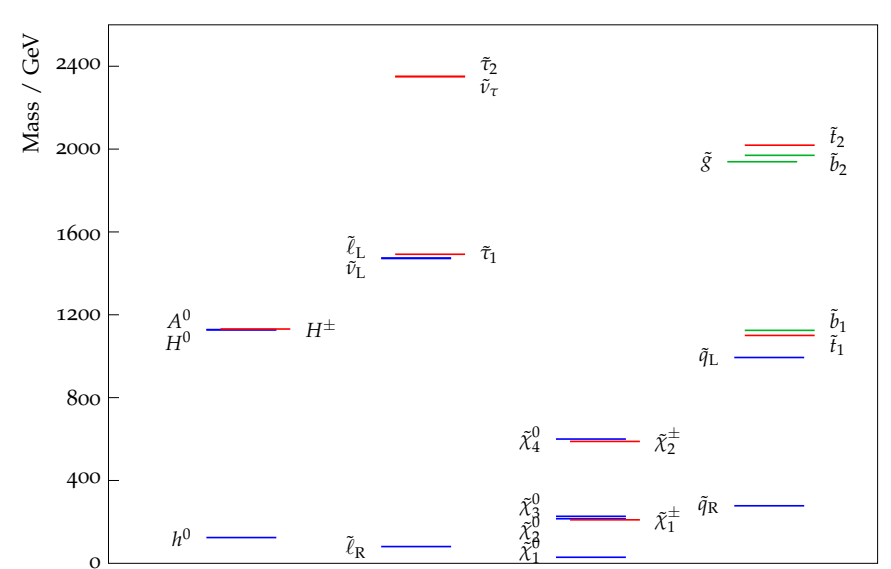

Fig. 5. Typical mass spectrum corresponding to the slepton NLSP scenario (class i) with $M_{\tilde{\chi}_{1}^{0}}=29.2 \mathrm{GeV}$ and $M_{\tilde{e}_{R}, \tilde{\mu}_{R}}=$ $80.1 \mathrm{GeV}$

gion which is inside the exclusion contours by the CDMS and XENON experiments.

We also check the cosmological constraints by considering the indirect detection constraints by Fermi-LAT [48]. The points passing all the constraints have neutralino annihilation cross-sections times relative velocity to $q \bar{q}$ smaller than $2 \times 10^{-30} \mathrm{~cm}^{3} / \mathrm{s}$, which is several orders of magnitude below the current Fermi-LAT limits and makes them compatible also with dark matter indirect detection limits.

\subsection{2 $\tilde{\chi}_{1}^{ \pm}$NLSP}

Next we consider points where the NSLP is the lightest chargino, $\chi_{1}^{ \pm}$and the $\Delta M$ mass splitting is small. This scenario corresponds generally to a compressed gaugino spectrum, with a small splitting between the chargino and neutralino masses. To accommodate the upper bound of the relic density constraint, a mass splitting of a few $\mathrm{GeV}$ between the lightest chargino and the lightest neutralino is generally required. As a consequence, the spin independent $\chi-p$ scattering cross-section is predicted to be relatively small, of order $10^{-6}-10^{-7} \mathrm{pb}$. Since this scenario has a small spin independent $\chi-p$ scattering cross-section, it is marginal in accommodating the claims for WIMP direct detection and corresponds to a region well inside the CDMS and XENON exclusion curves. Furthermore, the production cross-section of $\chi_{1}^{+} \chi_{1}^{-}$and $\chi_{2}^{0} \chi_{1}^{0}$ in this scenario at LEP-2 is large, 2.5 to $16 \mathrm{pb}$, and the detection efficiency of the LEP-2 analyses is $\sim 0.015-0.035$, corresponding to about 20 to 250 detected signal events. The cross-section upper limit from the combination of the data of the LEP-2 experiments 65] in this mass region is $\leq 1 \mathrm{pb}$ excluding all the points selected for this scenario. 

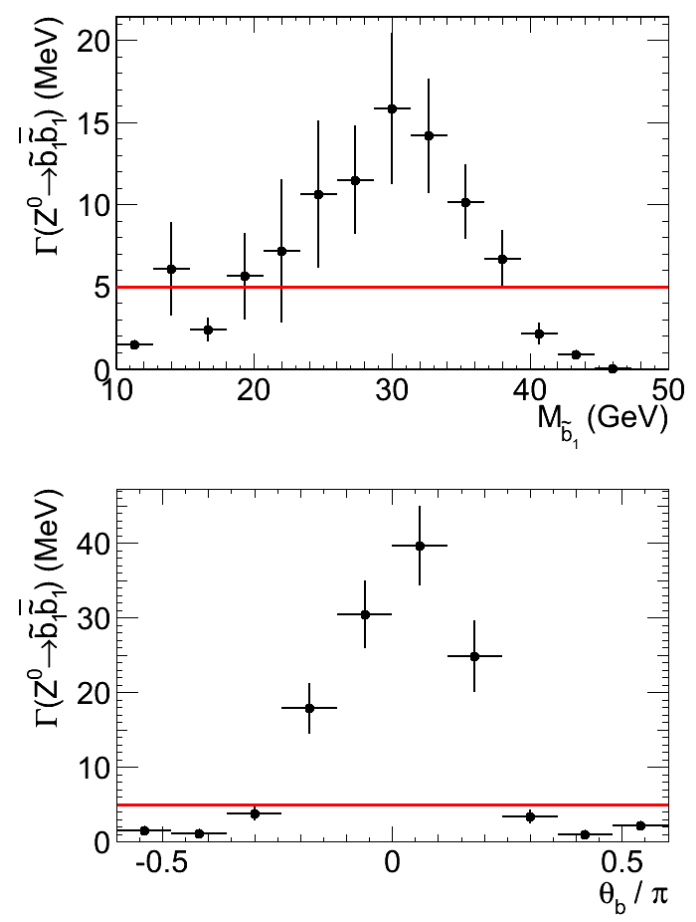

Fig. 6. Average $Z$ decay width to $\tilde{b}_{1} \overline{\tilde{b}}_{1}$ in function of the $\tilde{b}_{1}$ mass (upper panel) and the sbottom mixing angle $\theta_{b}$ (lower panel). The horizontal lines correspond to the experimental limit.

\subsubsection{Light $\tilde{q}_{L, R} \mathrm{NLSP}$}

We move over to the case of a scalar quark NLSP with small mass splitting with the lightest neutralino. The upper bound on the relic density constraint imposes a $\Delta M$ value of a few $\mathrm{GeV}$. The degenerate squark can be any of the squarks with the exception of the scalar top, since this is required to be heavier to accommodate the $h^{0}$ mass range highlighted by the LHC data. This scenario is the most interesting as it can provide us with a large spin independent $\chi-p$ scattering cross-section associated to a small neutralino mass (see Fig. 9). However, the couplings of the light squark to the $Z$ and $h^{0}$ bosons are in general large. In particular, the $Z$ decay width into squarks excludes this scenario, unless the squark decouples from the $Z$. This happens for specific values of the squark mixing angle. Since the first and second generation squarks do not mix, they are excluded leaving only a degenerate scalar bottom $\tilde{b}_{1}$ as a viable scenario.

The third generation left-handed squarks have a common mass in the pMSSM and a very light $\tilde{b}_{1}$ is only possible if the right-handed bottom squark is very light. In this case, the mixing angle $\theta_{b}$ is large, close to $\pi / 2$ corresponding to a mainly right-handed $\tilde{b}_{1}$, and the squark naturally decouples from the $Z$. The concurrence of a low value of the $\tilde{b}_{1}$ mass and its decoupling from the $Z$, through the mixing angle, is shown in Fig. 6. In addition, we observe that higher order SUSY corrections further decrease the $\tilde{b}_{1}$ mass, for appropriate values of the other param-

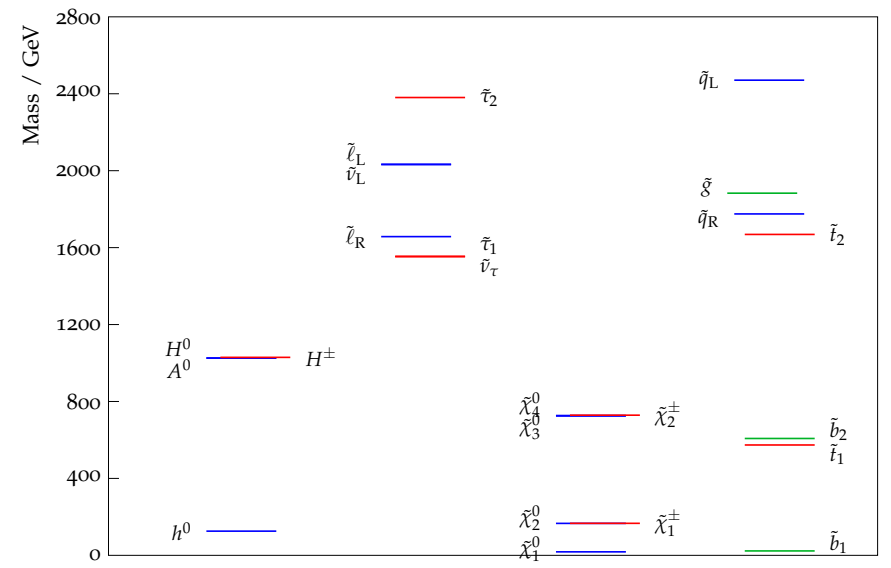

Fig. 7. Typical mass spectrum corresponding to the light sbottom degenerate with the lightest neutralino (class iii) with $M_{\tilde{\chi}_{1}^{0}}$ $=18.1 \mathrm{GeV}$ and $M_{\tilde{b}_{1}}=22.9 \mathrm{GeV}$

eters, producing typical spectra as that shown in Fig. 7 This "sbottom miracle" makes possible to find pMSSM solutions which reconcile a light neutralino signal at direct detection experiments with the LEP-1 constraints. At LEP-2 the pairs production of these light sbottom pairs has a cross-section of $\simeq 0.2 \mathrm{pb}$ and a selection efficiency of $0.15-0.40$. The LEP-2 data 66 excludes sbottom pair production with $\tilde{b} \rightarrow b \tilde{\chi}^{0}$ at cross-section values above $0.1 \mathrm{pb}$ in this mass region and therefore rejects points in this scenario unless the $\Delta M$ mass splitting between the $\tilde{b}_{1}$ and the $\tilde{\chi}_{1}^{0}$ is smaller, or of the order of, the $b$ quark mass, where the selection efficiency of the LEP-2 analyses drops. In most of cases, the decay $\tilde{b}_{1} \rightarrow \tilde{\chi}_{1}^{0} b$ is kinematically forbidden. At tree level, the most important open channel is $\tilde{b}_{1} \rightarrow \tilde{\chi}_{1}^{0} s$, which is CKM-suppressed and may increase the $\tilde{b}_{1}$ lifetime up to a value comparable to that of $b$ hadrons. Furthermore, large QCD corrections can be expected for such a light state, and decay channels with larger decay rate can open up at higher orders, thus decreasing the lifetime.

Considering now this light sbottom and the Higgs boson, we notice that the rate of the decay $h^{0} \rightarrow \tilde{b}_{1} \tilde{\tilde{b}}_{1}$ can become important in such scenarios. However, it is possible to find points for which the branching fraction of $h^{0} \rightarrow \tilde{b}_{1} \overline{\tilde{b}}_{1}$ and the $Z$ decay width to $\tilde{b}_{1} \overline{\tilde{b}}_{1}$ are simultaneously small. In Fig. 8, we show the correlations between the $Z$ decay width and the $h^{0}$ branching ratio to two photons. We remark that there is no strong correlations between both decays, and it is possible to have simultaneously a reduced $Z$ decay width to $\tilde{b}_{1} \overline{\tilde{b}}_{1}$ and very small $h^{0}$ branching fraction to $\tilde{b}_{1} \overline{\tilde{b}}_{1}$, even for $\tilde{b}_{1}$ masses as small as $15 \mathrm{GeV}$. Moreover, in this scenario, since the neutralino is mainly bino, the $h^{0}$ decaying to two light neutralinos is completely suppressed, resulting in an SM-like $h^{0}$ decay.

Finally, we check the cosmological constraints by considering the indirect detection constraints by Fermi-LAT [48. The selected points corresponding to the degener- 


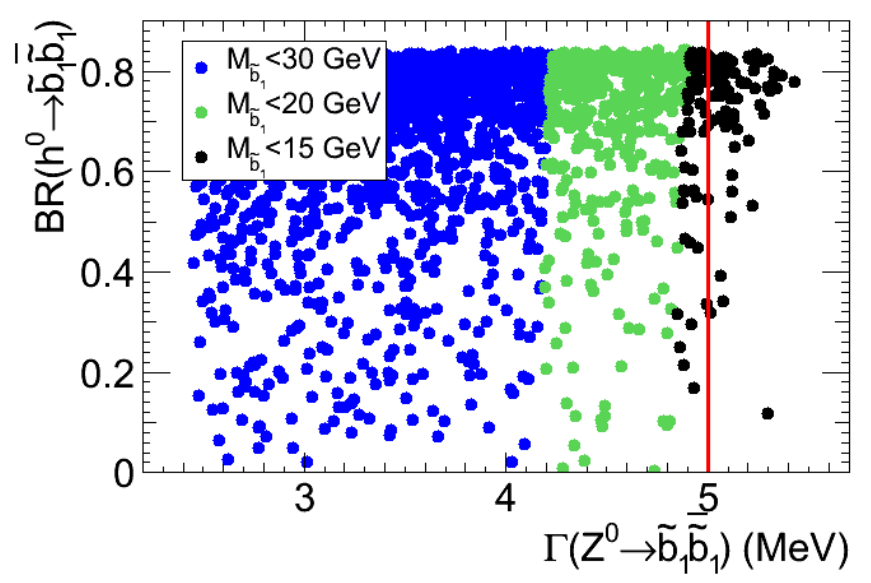

Fig. 8. $h^{0}$ branching fraction to $\tilde{b}_{1} \overline{\tilde{b}}_{1}$ as a function of the $Z$ decay width to $\tilde{b}_{1} \overline{\tilde{b}}_{1}$ for different values of the lightest sbottom mass.

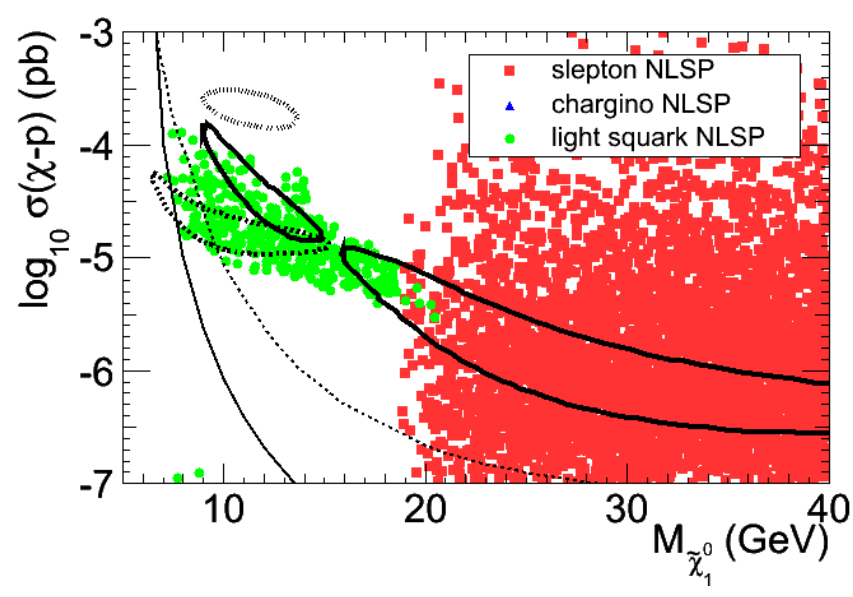

Fig. 9. Spin independent $\chi$-p scattering cross-section as a function of the $\tilde{\chi}_{1}^{0}$ mass. The points presented here pass all the previous constraints, including the tight relic density bounds. The red squares correspond to a slepton NLSP with a mass slightly above the LEP limits (class i), the blue triangles to scenarios with a chargino NLSP (class ii), and the green points to cases where a scalar quark is degenerate with the light neutralino (class iii).

ate $\tilde{b}_{1}$ scenario have neutralino annihilation cross-sections times relative velocity to $b \bar{b}$ smaller than $5 \times 10^{-27} \mathrm{~cm}^{3} / \mathrm{s}$, which is one order of magnitude below the current FermiLAT limits, which makes them compatible also with dark matter indirect detection limits.

In summary, after considering the constraint from the LEP data, the only viable scenario with a neutralino mass below $20 \mathrm{GeV}$ corresponds to the light sbottom NLSP case.

In Fig. 9, we present distribution of the points passing the tight relic density bound. Alternatively, in Fig. 10, the same distribution is presented in the case where the loose relic density constraint is used.

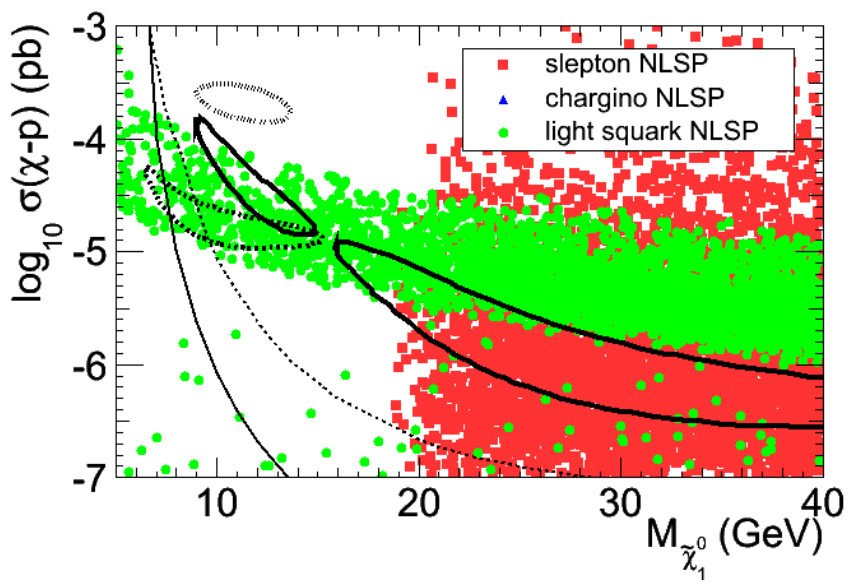

Fig. 10. Spin independent $\chi$-p scattering cross-section as a function of the $\tilde{\chi}_{1}^{0}$ mass. The points presented here pass all the previous constraints, including the loose relic density bound. The red squares correspond to a slepton NLSP with a mass slightly above the LEP limits (class i), the blue triangles to scenarios with a chargino NLSP (class ii), and the green points to cases where a scalar quark is degenerate with the light neutralino (class iii).

A comparison of these two figures reveals that the lower bound of the relic density reduces the overall statistics, but also removes points corresponding to scenarios with a scalar quark degenerate with the light neutralino for neutralino masses above $20 \mathrm{GeV}$. This can be explained by the fact that points with a very small relic density have a small splitting. However, to get a relic density in the WMAP interval, the splitting should not be too small relatively to the neutralino mass. Also, the direct search bounds disfavour large splittings. Therefore, combining the relic density and direct search limits, only a small window remains where points can pass all the constraints.

\subsection{Non-standard scenarios}

The calculation of the relic density and the dark matter direct detection constraints rely on many assumptions. In particular, different cosmological scenarios can lead to a relic density which is larger than that computed in the standard cosmological scenario. First, the neutralino could be only one of several dark matter components. Then, if dark energy were the dominant component at the time of the relic freeze-out, it would result in an acceleration of the expansion of the Universe, which would lead to an earlier freeze-out and a much larger relic density $67-71$. Finally, entropy generation at the time of freeze-out, for example due to the decay of a late inflaton, can also lead to an increase - or a decrease - of the relic density [72 76 . These effects are however limited by Big-Bang nucleosynthesis constraints, but using AlterBBN [77, we verified that they can nevertheless lead to an increase of three or- 


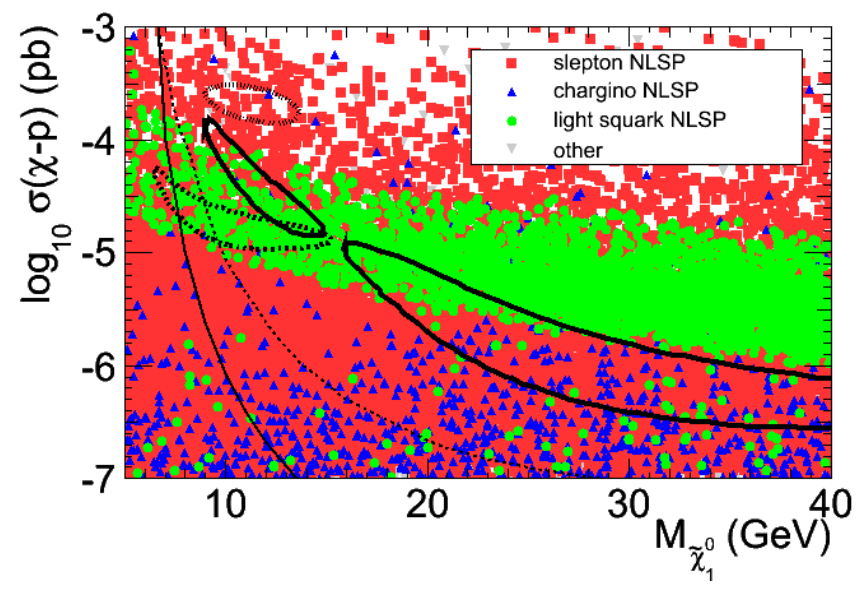

Fig. 11. Spin independent $\chi$-p scattering cross-section in function of the $\tilde{\chi}_{1}^{0}$ mass. The points presented here pass all the previous constraints, without applying the relic density limits. The red points correspond to a slepton NLSP with a mass slightly above the LEP limits (class i), the blue points to scenarios with a chargino NLSP (class ii), the green points to cases where a scalar quark is degenerate with the light neutralino (class iii), and the gray point to other more canonical scenarios.

ders of magnitudes or more of the relic density while still being compatible with BBN constraints.

Similarly to the relic density constraint, the direct detection constraints also rely on several assumptions. In particular, if the neutralino is not the only component of dark matter, or if the local density or velocity of dark matter is widely different from the standard assumptions ${ }^{1}$ the constraints in terms of scattering cross-sections can be drastically changed. Therefore, even if the detection of a WIMP particles by a detector would permit to fix its mass, its scattering cross-section with matter would be dependent on large astrophysical uncertainties. The monojet searches at colliders however set constraints on the scattering cross-sections without suffering from astrophysical uncertainties.

With these considerations, it is worth considering the results that we obtain by relaxing the relic density constraint. Fig. 11 shows the different classes of points which pass all the constraints, without applying the relic density limits. The three classes described above are well represented. In particular, the scenarios with degenerate squarks and sleptons are now realised over a broader range of scattering cross-sections and light neutralino mass values. In addition, the chargino NLSP scenario is allowed in this case. In particular, the relic density constraint imposes a small splitting between the neutralino and the NLSP. With this constraint removed, the splitting condition is strongly weakened, and it becomes possible to find compressed gaugino scenarios which pass LEP-2 $\chi_{1}^{+} \chi_{1}^{-}$ and $\chi_{2}^{0} \chi_{1}^{0}$ production constraint. Therefore these two classes

${ }^{1}$ See for example [78] for a discussion about the local density of dark matter.
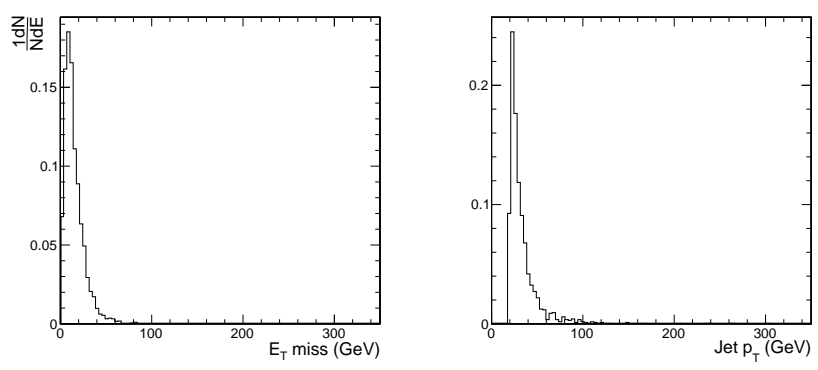

Fig. 12. Distributions of event missing $E_{T}$ (left) and jet $p_{T}$ (right) for $\tilde{b}_{1}$ production in $8 \mathrm{TeV} p p$ collisions in the almost degenerate light $\tilde{b}_{1}$ NLSP scenario.

of spectra can be rehabilitated if the relic density constraint is relaxed.

We also notice that other points which do not belong to any of the three classes can fulfill all the conditions. These scenarios have no other peculiarity than having a light neutralino and a scalar particle with a mass of a few hundreds of $\mathrm{GeV}$, which can increase the scattering cross-sections.

\section{Sensitivity at LHC}

In general terms, MSSM scenarios with a light neutralino offer no specific challenges to the LHC searches. Limits for $\tilde{g}$ and $\tilde{q}$ masses are commonly reported in the $M_{\chi_{1}^{0}}=$ 0 limit. What makes the most viable scenario identified in this study specific for their search at the LHC is the high level of mass degeneracy between the LSP $\tilde{\chi}_{1}^{0}$ and the $\tilde{b}_{1}$ scalar quark. This implies a very large production crosssection, of order $0.6 \mu \mathrm{b}$ at $8 \mathrm{TeV}$ accompanied by events with small transverse energy.

We study the distribution of the observables employed in the MET SUSY searches for a few points belonging to the various scenarios identified above. Here a word of caution is in order, since this analysis is carried out using fast simulation for events with a remarkably different kinematics compared to those used for its validation. Results can be considered valid in broader, qualitative terms but not necessary quantitatively. Fig. 12 shows the distributions we obtain for the event missing $E_{T}$ and the jet $p_{T}$ for one of the selected points in the almost degenerate light $\tilde{b}_{1}$ NLSP scenario, which are relevant for the trigger and selection cut applied in MET analyses. By applying the jet $p_{T}$ and event missing $E_{T}$ cuts adopted in the recent searches for scalar bottom quark pair production with the ATLAS detector [79, 80, from a sample of 10k no events are kept. Similar strong cuts on missing $E_{T}$ and jet $p_{T}$ were applied in an earlier CMS search 81. However, if the cuts on the two variables would be lowered from $130 \mathrm{GeV}$ to 75 and $50 \mathrm{GeV}$, the selection efficiency would become of the order of 0.003 and 0.012 , respectively. 


\section{Conclusions}

While the LHC experiments are progressively testing the SUSY parameter space for possible signals of stronglyinteracting supersymmetric particle partners and they report events compatible with a Higgs signal, ground-based dark matter direct detection experiments offer a complementary approach in the search for a new weakly-interacting particle, which can be identified with the SUSY lightest neutralino. Three independent experiments have reported possible signals, which may be interpreted as due to the interaction of relic light neutralinos exhibiting a large scattering cross section, in their detectors. In this paper we have investigated the compatibility of such signals with the generic minimal supersymmetric extension of the Standard Model, taking into account the constraints from the low energy, flavour, LEP and LHC data. Through high statistics flat scans of the pMSSM we have identified several scenarios which give rise to very light neutralinos with large scattering cross section. Once the LEP and LEP-2 limits are taken into account, the only viable scenario has the lightest scalar bottom quark, $\tilde{b}_{1}$ almost degenerate with the neutralino. This is compatible with the dark matter relic density from WMAP and indirect constraints from Fermi-LAT. A sizeable fraction of these points correspond to interaction cross section values close to the present constraints from CDMS and XENON. Relaxing the constraints from WMAP on the other hand leaves room also for other classes of scenarios such as the chargino or slepton NLSP cases to manifest themselves.

\section{Acknowledgements}

We would like to thank M. Mangano for supporting this activity and the LPCC for making dedicated computing resources available to us. We are thankful to $\mathrm{Ka} \mathrm{Ki} \mathrm{Li}$ for her contribution during the early stage of this study. We acknowledge discussions with B. Allanach, A. Djouadi, A. De Roeck, G. Bélanger, E. Nezri, G. Polesello, K. Rolbiecki, T. Riemann, M. Spira, E. Aprile, P. Beltrame, A. Melgarejo and D. Speller. We are also grateful to E. Gianolio and the CERN IT Department for computing support.

\section{References}

1. E. Aprile, et al. (XENON 100 Collaboration), Phys. Rev. Lett. (2011), arXiv:1104.2549.

2. Z. Ahmed, et al. (The CDMS-II Collaboration), Science 327, 1619 (2010), arXiv:0912.3592.

3. A. Arbey, M. Battaglia, F. Mahmoudi, Eur. Phys. J. C72, 1906 (2012), arXiv:1112.3032.

4. R. Bernabei, et al. (DAMA Collaboration), Phys. Lett. B480, 23 (2000)

5. R. Bernabei, P. Belli, F. Cappella, et al., Eur. Phys. J. C67, 39 (2010), arXiv:1002.1028.

6. C. Aalseth, et al. (CoGeNT collaboration), Phys. Rev. Lett. 106, 131301 (2011), arXiv:1002.4703.
7. C. Aalseth, P. Barbeau, J. Colaresi, et al., Phys. Rev. Lett. 107, 141301 (2011), arXiv:1106.0650.

8. G. Angloher, M. Bauer, I. Bavykina, et al. (2011), arXiv:1109.0702.

9. C. Savage, G. Gelmini, P. Gondolo, et al., JCAP 0904, 010 (2009), arXiv:0808.3607.

10. A. Fitzpatrick, D. Hooper, K. M. Zurek, Phys. Rev. D81, 115005 (2010), arXiv:1003.0014.

11. C. Kelso, D. Hooper, M. R. Buckley (2011), arXiv: 1110.5338 .

12. G. B. Gelmini (2012), arXiv:1201.4560.

13. A. Djouadi, et al. (MSSM Working Group) (1998), hep-ph/9901246.

14. D. Hooper, T. Plehn, Phys. Lett. B562, 18 (2003), hep-ph/0212226.

15. A. Bottino, N. Fornengo, S. Scopel, Phys. Rev. D67, 063519 (2003), hep-ph/0212379.

16. A. Bottino, F. Donato, N. Fornengo, et al., Phys. Rev. D69, 037302 (2004), hep-ph/0307303.

17. S. Profumo, Phys. Rev. D78, 023507 (2008), arXiv: 0806.2150 .

18. A. Bottino, N. Fornengo, G. Polesello, et al., Phys. Rev. D77, 115026 (2008), arXiv:0801.3334.

19. E. Dudas, S. Lavignac, J. Parmentier, Nucl. Phys. B808, 237 (2009), arXiv:0808.0562.

20. D. Feldman, Z. Liu, P. Nath, Phys. Rev. D81, 117701 (2010), arXiv: 1003.0437.

21. E. Kuflik, A. Pierce, K. M. Zurek, Phys. Rev. D81, 111701 (2010), arXiv:1003.0682.

22. A. V. Belikov, J. F. Gunion, D. Hooper, et al., Phys. Lett. B705, 82 (2011), arXiv:1009.0549.

23. D. A. Vasquez, G. Belanger, C. Boehm, et al., Phys. Rev. D82, 115027 (2010), arXiv:1009.4380.

24. S. Choi, S. Scopel, N. Fornengo, et al., Phys. Rev. D85, 035009 (2012), arXiv:1108.2190.

25. D. Albornoz Vasquez, G. Belanger, C. Boehm, Phys. Rev. D84, 095015 (2011), arXiv:1108.1338.

26. N. Bhattacharyya, A. Choudhury, A. Datta (2011), arXiv:1107.1997.

27. D. T. Cumberbatch, D. E. Lopez-Fogliani, L. Roszkowski, et al. (2011), arXiv:1107.1604.

28. L. Calibbi, T. Ota, Y. Takanishi, JHEP 1107, 013 (2011), arXiv: 1104.1134.

29. A. Bottino, N. Fornengo, S. Scopel (2011), arXiv: 1112.5666 .

30. L. Calibbi, T. Ota, Y. Takanishi (2011), arXiv:1112. 0219.

31. V. Barger, P. Langacker, H.-S. Lee, Phys. Lett. B630, 85 (2005), hep-ph/0508027.

32. J. F. Gunion, D. Hooper, B. McElrath, Phys. Rev. D73, 015011 (2006), hep-ph/0509024.

33. D. Das, U. Ellwanger, JHEP 1009, 085 (2010), arXiv:1007.1151.

34. J. F. Gunion, A. V. Belikov, D. Hooper (2010), arXiv: 1009.2555.

35. M. Carena, N. R. Shah, C. E. Wagner, Phys. Rev. D85, 036003 (2012), arXiv:1110.4378.

36. A. Arbey, M. Battaglia, F. Mahmoudi, Eur. Phys. J. C72, 1847 (2012), arXiv:1110.3726. 
37. B. C. Allanach, Comput. Phys. Commun. 143, 305 (2002), hep-ph/0104145.

38. M. Muhlleitner, A. Djouadi, Y. Mambrini, Comput. Phys. Commun. 168, 46 (2005), hep-ph/0311167.

39. F. Mahmoudi, Comput. Phys. Commun. 178, 745 (2008), arXiv:0710.2067;

F. Mahmoudi, Comput. Phys. Commun. 180, 1579 (2009), arXiv:0808.3144.

40. A. Arbey, F. Mahmoudi, Comput. Phys. Commun. 181, 1277 (2010), arXiv:0906.0369.

41. G. Belanger, F. Boudjema, A. Pukhov, et al., Comput. Phys. Commun. 180, 747 (2009), arXiv:0803.2360.

42. T. Sjostrand, S. Mrenna, P. Z. Skands, JHEP 05, 026 (2006), hep-ph/0603175.

43. W. Beenakker, R. Hopker, M. Spira (1996), hep-ph/ 9611232.

44. S. Ovyn, X. Rouby, V. Lemaitre (2009), arXiv: 0903. 2225.

45. E. Komatsu, et al. (WMAP Collaboration), Astrophys. J. Suppl. 192, 18 (2011), arXiv:1001.4538.

46. Z. Ahmed, et al. (CDMS-II Collaboration), Phys. Rev. Lett. 106, 131302 (2011), arXiv:1011.2482.

47. Z. Ahmed, et al. (CDMS Collaboration) (2012), arXiv:1203.1309.

48. M. Ackermann, et al. (Fermi-LAT collaboration), Phys. Rev. Lett. 107, 241302 (2011), arXiv:1108. 3546.

49. A. Akeroyd, F. Mahmoudi, D. Martinez Santos, JHEP 1112, 088 (2011), arXiv: 1108.3018.

50. T. Aaltonen, et al. (CDF), Phys. Rev. Lett. 107, 239903 (2011), arXiv:1107.2304.

51. R. Aaij, et al. (LHCb collaboration) (2012), arXiv: 1203.4493.

52. S. Chatrchyan, et al. (CMS Collaboration) (2012), arXiv:1203.3976.

53. F. Mahmoudi, S. Neshatpour, J. Orloff (2012), arXiv: 1205.1845.

54. D. Y. Bardin, P. Christova, M. Jack, et al., Comput. Phys. Commun. 133, 229 (2001), hep-ph/9908433.

55. J. Abdallah, et al. (DELPHI), Eur. Phys. J. C31, 421 (2003), hep-ex/0311019.

56. G. Aad, et al. (ATLAS Collaboration), Phys. Lett. B710, 67 (2012), arXiv:1109.6572.

57. S. Chatrchyan, et al. (CMS Collaboration), Phys. Rev. Lett. 107, 221804 (2011), arXiv:1109.2352.

58. G. Aad, et al. (ATLAS Collaboration), Phys. Lett. B705, 174 (2011), arXiv:1107.5003.

59. S. Chatrchyan, et al. (CMS Collaboration) (2012), arXiv:1202.4083.

60. G. Aad, et al. (ATLAS Collaboration), Phys. Lett. B710, 49 (2012), arXiv: 1202.1408.

61. S. Chatrchyan, et al. (CMS Collaboration), Phys. Lett. B710, 26 (2012), arXiv:1202.1488.

62. A. Arbey, M. Battaglia, A. Djouadi, et al., Phys. Lett. B708, 162 (2012), arXiv:1112.3028.

63. S. Chatrchyan, et al. (CMS Collaboration) (2012), arXiv:1204.0821.

64. Y. Bai, P. J. Fox, R. Harnik, JHEP 1012, 048 (2010), arXiv:1005.3797.
65. LEP2 SUSY Working Group (2002), NoteLEPSUSYWG/ 02-04.1.

66. LEP2 SUSY Working Group (2004), NoteLEPSUSYWG/ 04-02.1.

67. M. Kamionkowski, M. S. Turner, Phys. Rev. D42, 3310 (1990)

68. P. Salati, Phys. Lett. B571, 121 (2003), astro-ph/ 0207396.

69. S. Profumo, P. Ullio, JCAP 0311, 006 (2003), hep-ph/0309220.

70. D. J. Chung, L. L. Everett, K. Kong, et al., JHEP 0710, 016 (2007), arXiv:0706.2375.

71. A. Arbey, F. Mahmoudi, Phys. Lett. B669, 46 (2008), arXiv:0803.0741.

72. T. Moroi, L. Randall, Nucl. Phys. B570, 455 (2000), hep-ph/9906527.

73. G. F. Giudice, E. W. Kolb, A. Riotto, Phys. Rev. D64, 023508 (2001), hep-ph/0005123.

74. N. Fornengo, A. Riotto, S. Scopel, Phys. Rev. D67, 023514 (2003), hep-ph/0208072.

75. G. Gelmini, P. Gondolo, A. Soldatenko, et al., Phys. Rev. D74, 083514 (2006), hep-ph/0605016.

76. A. Arbey, F. Mahmoudi, JHEP 1005, 051 (2010), arXiv:0906.0368.

77. A. Arbey, Comput. Phys. Commun. 183, 1822 (2012), arXiv:1106.1363.

78. C. M. Bidin, G. Carraro, R. Mendez, et al. (2012), arXiv:1204.3924.

79. G. Aad, et al. (ATLAS Collaboration), Phys. Rev. Lett. 108, 181802 (2012), arXiv:1112.3832.

80. G. Aad, et al. (ATLAS Collaboration) (2012), arXiv: 1203.6193.

81. CMS Collaboration (2011), CMS-PAS-SUS-11-006. 